\title{
Post-retrieval $\boldsymbol{\beta}$-adrenergic receptor blockade: Effects on extinction and reconsolidation of cocaine-cue memories
}

\author{
Ashley N. Fricks-Gleason and John F. Marshall' \\ Department of Neurobiology and Behavior, Center for the Neurobiology of Learning and Memory, University of California, \\ Irvine, California 92697, USA
}

\begin{abstract}
Contexts and discrete cues associated with drug-taking are often responsible for relapse among addicts. Animal models have shown that interference with the reconsolidation of drug-cue memories can reduce seeking of drugs or drug-paired stimuli. One such model is conditioned place preference (CPP) in which an animal is trained to associate a particular environment with the rewarding effects of a drug. Previous work from this laboratory has shown that intra-nucleus accumbens core infusions of a MEK inhibitor can interfere with reconsolidation of these drug-cue memories. A question that remains is whether post-retrieval drug effects on subsequent memories represent an interference with reconsolidation processes or rather a facilitation of extinction. In this experiment, we explore the effect of post-retrieval injections of propranolol, a $\boldsymbol{\beta}$-adrenergic receptor antagonist, on reconsolidation and extinction of cocaine CPP. After acquisition of cocaine CPP, animals were given post-retrieval propranolol injections once or each day during a protocol of unreinforced preference tests, until the animals showed no preference for the previously cocaine-paired environment. Following a cocaine priming injection, the animals that received daily post-test propranolol injections did not reinstate their preference for the drug-paired side. In contrast, a single post-retrieval propranolol injection followed by multiple days of unreinforced preference tests failed to blunt subsequent cocaine reinstatement of the memory. These data suggest that daily post-retrieval systemic injections of propranolol decrease the conditioned preference by interfering with reconsolidation of the memory for the association between the drug-paired side and the reinforcing effects of the drug, rather than facilitating new extinction learning.
\end{abstract}

For addicts, relapse into drug seeking is often triggered by drugpaired cues. During episodes of drug taking, associations are formed between previously neutral cues and the rewarding effects of the drug. These environmental cues take on incentive motivational properties that can lead to intense craving when encountered by addicts (Childress et al. 1988), even after long periods of abstinence. Recent interest has focused on the possibility that these memories can be attenuated, potentially leading to novel treatments for addiction and other neuropsychiatric disorders. Animal models allow for a detailed understanding of the molecular and systems-level processes underlying cue-elicited drug seeking, and as such have proven valuable in identifying potential treatments.

The reconsolidation hypothesis posits that memories become labile when activated via recall and are subsequently reconsolidated. Although early studies suggested that the processes for consolidation and reconsolidation used the same mechanisms (Sara 2000), recent evidence has pointed to divergent processes (Taubenfeld et al. 2001; Lee et al. 2004). Most of the evidence for reconsolidation has been generated using fear conditioning paradigms. Only recently has reconsolidation been investigated in appetitive paradigms, including drug selfadministration and conditioned place preference (CPP). In both fear-motivated and appetitive learning paradigms, memory disruption during the labile phase leads to decreased performance in subsequent tests (Lee et al. 2005; Miller and Marshall 2005; Bernardi et al. 2006; Diergaarde et al. 2006). Using a self-

\footnotetext{
1Corresponding author.
}

E-mail jfmarsha@uci.edu; fax (949) 824-2447.

Article is online at http://www.learnmem.org/cgi/doi/10.1101//m.1054608. administration paradigm, Lee et al. (2005) demonstrated that a conditioned stimulus associated with cocaine (COC) could support the development of a new instrumental drug-seeking response. Furthermore, they were able to disrupt reconsolidation of this drug-cue memory via intra-amygdalar infusion of Zif268 antisense oligonucleotides. Using cocaine CPP, Miller and Marshall (2005) found that intra-accumbens infusions of an inhibitor of mitogen-activated protein kinase (MAP kinase) blocked reconsolidation of cocaine-contextual cue memories and interfered with retrieval-dependent activation of the MAP kinase intracellular signaling cascade. The results of these two studies indicate that drug memories undergo reconsolidation in a manner similar to fear memories (Duvarci and Nader 2004). Subsequent studies showed that post-retrieval systemic administration of compounds that act at neurotransmitter receptors, not just those acting on intracellular signaling pathways, can interfere with cocaine-cue associations. Using a cocaine CPP paradigm, Bernardi et al. (2006) demonstrated that systemic administration of propranolol, a $\beta$-adrenergic receptor antagonist, given immediately post-retrieval, disrupted reconsolidation of drug-context memories.

The mechanism by which these experimental treatments disrupt drug-cue associations is uncertain. They may interfere with memory reconsolidation or instead may work through other associative processes, e.g., by facilitating extinction for the learned behavior. Traditionally, extinction refers to the process during which repeated nonreinforced presentations of a conditioned stimulus (CS) result in decreased amplitude and frequency of the conditioned response (Pavlov 1927). Extinction does not represent "unlearning" of the original association between CS and unconditioned stimulus, but instead results from new learn- 
ing that counteracts the effects of the conditioned response (for reviews, see Myers and Davis 2002; Delamater 2004). In experimental paradigms related to drug reinforcement, extinction learning can be expected to decrease response to the drug-paired stimuli but not to induce loss of the original association. After acquisition and subsequent extinction of either drug-based selfadministration or CPP, responses for drug-paired cues can be reinstated, for example, by a single injection of the drug prior to testing ("priming"; Stewart 1983; Mueller and Stewart 2000). While experimental interventions that either facilitate extinction or interfere with reconsolidation of drug-cue memories may both contribute to the therapeutics of addiction, successfully distinguishing between these differing effects should greatly help elucidate the processes underlying the resiliency of these memories.

The present study investigated the effect of systemic postretrieval injections of propranolol on the reconsolidation and extinction of cocaine CPP memories. We show that post-retrieval administration of propranolol, depending on the pattern of administration, can have two effects on the cocaine-context associations in CPP. A single post-retrieval dose of propranolol, followed by multiple days of extinction training, resulted in animals' reaching extinction criteria sooner but did not diminish subsequent reinstatement by a cocaine priming injection. In contrast, multiple daily post-retrieval injections of propranolol both accelerated the rate at which preference was lost and eliminated subsequent cocaine reinstatement, findings consistent with reconsolidation interference.

\section{Results}

\section{Experiment 1: Single post-retrieval propranolol administration}

Following CPP conditioning, animals spent significantly more time in the drug-paired compartment relative to the drugunpaired compartment (Wilcoxon test, $z=-5.74, P<0.001$ ). Similar significant preferences were preserved when the animals were divided into treatment groups (Wilcoxon tests, prospective propranolol group: $Z=-3.98, P<0.001$; prospective saline group: $Z=-4.17, P<0.001)$. These findings demonstrate the development of a preference for the environment previously paired with COC. Injection of propranolol immediately after post-test 1 reduced but did not abolish the subsequent preference for the drug-paired compartment compared to saline-injected controls. The propranolol group retained a significant preference for the drug-paired compartment at post-test 2 (Wilcoxon test, $Z=-3.95, P<0.001$ ) yet, compared to post-test 1 , spent significantly less time in the drug-paired compartment (Wilcoxon test, $Z=-3.18, P<0.001$ ) (Fig. 1A). The saline animals retained their preference for the drug-paired compartment at post-test 2 (Wilcoxon test, $Z=-4.20, P<0.001)$, spending a comparable amount of time in the drug-paired compartment relative to posttest 1 (Fig. 1B).

During subsequent daily unreinforced preference testing, the group that received propranolol after post-test 1 reached the no-preference criterion sooner than the saline group. By post-test 8 , the propranolol animals had met the criterion of two consecutive days with no significant preference for the drug-paired compartment and a $<90$-sec difference in time spent in each compartment (Fig. 1A). The saline animals, however, did not meet this criterion until post-test 10 (Fig. 1B). There was neither a significant main effect of treatment group nor a treatment-byday interaction.

In response to the cocaine priming injection, both treat-

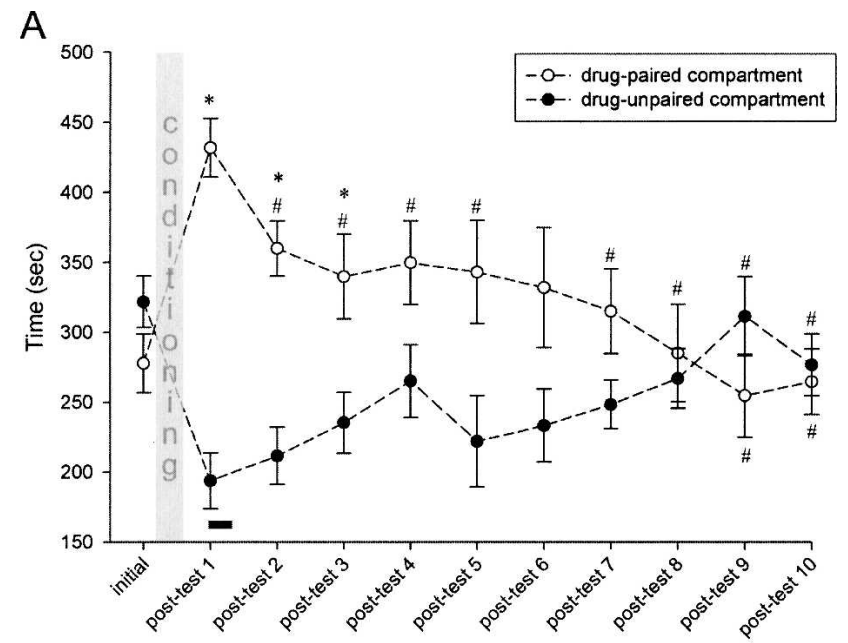

B
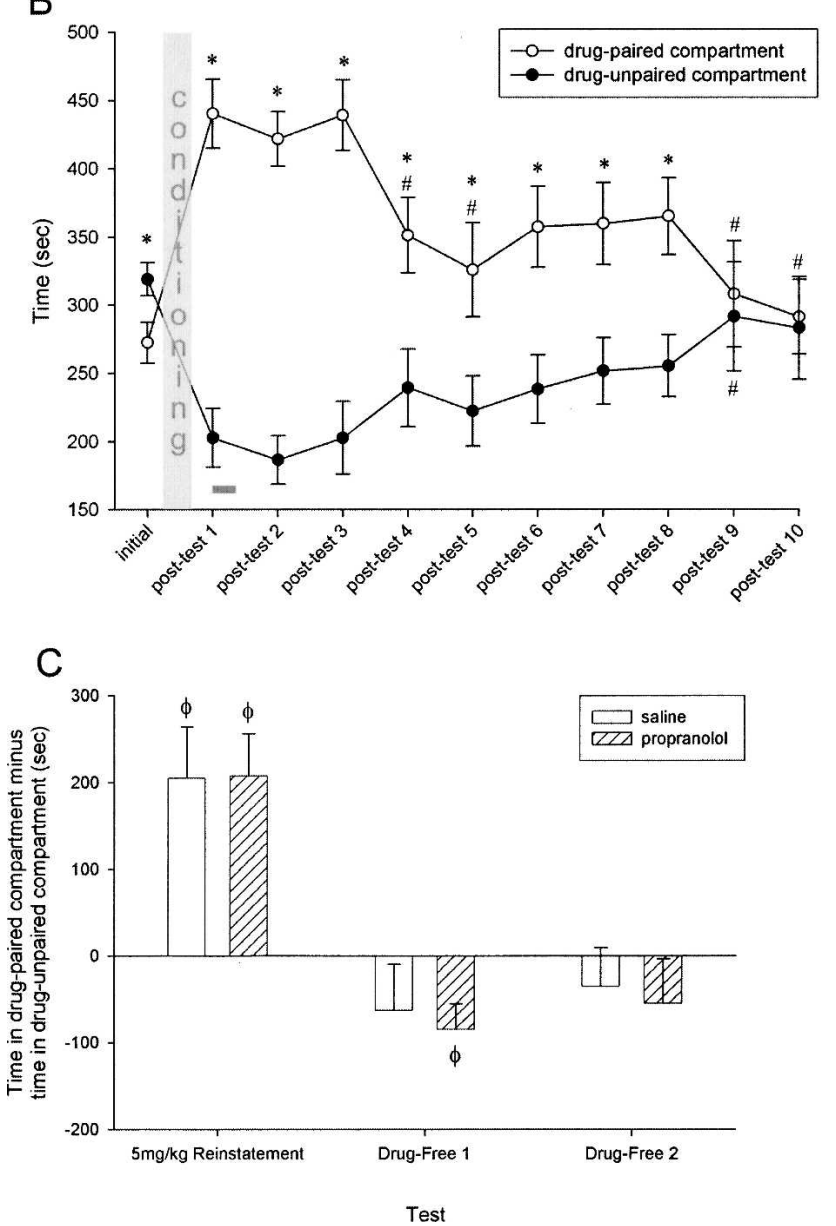

Figure 1. $(A, B)$ COC-CPP expression indicated by mean \pm S.E.M. time spent in the drug-paired vs. drug-unpaired compartments. (A) Propranolol group $(n=10)$, (B) saline group $(n=12)$. Black bar indicates occurrence of propranolol injection; gray bar indicates occurrence of saline injection. $\left(^{*}\right)$ Indicates significant difference between compartments, Wilcoxon tests, $P<0.05$. (\#) Indicates significant difference from post-test 1 , Wilcoxon tests, $P<0.05$. (C) COC-CPP expression following reinstatement indicated by time in the drug-paired compartment minus time in the drug-unpaired compartment. $(\Phi)$ Indicates significant difference from zero, one-sample $t$-tests, $P<0.05$. 
ment groups robustly reinstated their CPP, exhibiting a significant preference for the drug-paired compartment (one-sample $t$-tests, propranolol group: $t=4.216, P<0.01$; saline group: $t=3.486, P<0.01)$. This preference can be represented as a difference score, calculated as the time spent in the drug-paired compartment minus the time spent in the drug-unpaired compartment, which was compared against zero to determine the level of preference. When subsequently tested on each of two days in a drug-free state, neither treatment group expressed a preference. Both the propranolol animals and the saline animals had significantly lower difference scores at drug-free test 1 (Wilcoxon tests, propranolol group: $Z=-2.80, P<0.01$; saline group: $Z=-2.82, P<0.01$ ) and drug-free test 2 (Wilcoxon tests, propranolol group: $Z=-2.29, P<0.05$; saline group: $Z=-2.90$, $P<0.01)$ relative to the $5-\mathrm{mg} / \mathrm{kg}$ drug-primed reinstatement test (Fig. 1C).

\section{Experiment 2: Multiple post-retrieval propranolol administrations}

Following CPP conditioning, animals spent significantly more time in the drug-paired compartment relative to the drugunpaired compartment (Wilcoxon test, $z=-4.29, P<0.001$ ). Similar significant preferences were preserved when the animals were divided into treatment groups (Wilcoxon tests, prospective propranolol group: $z=-3.06, P<0.01$; prospective saline group: $Z=-3.06, P<0.01)$. These findings demonstrate the development of a preference for the environment previously paired with COC.

Similar to the results in experiment 1 , the injection of propranolol immediately after post-test 1 reduced but did not abolish the preference for the drug-paired compartment relative to saline-injected controls (Fig. 2A,B). On post-test 2, both the propranolol and saline groups retained their preference for the drugpaired compartment (Wilcoxon tests, propranolol group: $z=-2.98, P<0.01$; saline group: $z=-2.90, P<0.01$ ), although the propranolol animals did exhibit a mildly decreased preference for the drug-paired compartment.

During subsequent unreinforced preference training, in which injections of propranolol or saline were given after each daily test, the propranolol group reached the no-preference criterion much sooner than the saline group. By post-test 7 , the propranolol animals had met the criterion of two consecutive days with no significant preference for the drug-paired compartment and a $<90$-sec difference in time spent in each compartment (Fig. 2A). The saline animals, by comparison, did not reach this criterion until post-test 12 (Fig. 2B). When comparing the difference scores, calculated as the time spent in the drug-paired compartment minus the time spent in the drug-unpaired compartment, over the $13 \mathrm{~d}$ of preference testing, there was a significant main effect of treatment group (general linear model, $F=5.98, P<0.05)$ as well as a significant treatment-by-day interaction (general linear model, $F=1.95, P<0.05$ ).

In response to the first cocaine priming injection, the saline group robustly reinstated, as evidenced by a difference score that was significantly different from zero (one-sample $t$-test, $t=6.021$, $P<0.001$ ). In contrast to experiment 1 , the propranolol group did not show significant reinstatement following the $5-\mathrm{mg} / \mathrm{kg}$ cocaine priming injection, as evidenced by a difference score that was not different from zero and was significantly lower than that of the saline group (ANOVA, $F=4.45, P<0.05$ ). When tested in a drug-free state the following day, neither treatment group expressed a preference, having difference scores that were significantly lower than those at the $5-\mathrm{mg} / \mathrm{kg}$ drug-primed reinstatement test (Wilcoxon tests, propranolol group: $z=-1.96$, $P<0.05$; saline group: $Z=-2.98, P<0.01)$. In response to a lower dose $(2.5 \mathrm{mg} / \mathrm{kg})$ cocaine priming injection, the saline group again showed a significant reinstatement of preference (one-sample $t$-test, $t=3.849, P<0.01$ ), but the magnitude of this preference was significantly lower than that induced by the higher dose priming injection (Wilcoxon test, $z=-2.28$, $P<0.05)$. The propranolol group expressed no preference in response to this same $2.5-\mathrm{mg} / \mathrm{kg}$ cocaine priming injection. Similar to the results of the first drug-free test, both groups expressed no preference at drug-free tests 2 and 3. Finally, in response to a second low $(2.5 \mathrm{mg} / \mathrm{kg})$ dose cocaine priming injection, the saline animals exhibited a significant preference for the drugpaired compartment (one-sample $t$-test, $t=4.621, P=0.001$ ), reinstating to a level comparable to that seen following the first $2.5-\mathrm{mg} / \mathrm{kg}$ priming injection. At this final drug-primed reinstatement test, the propranolol animals again exhibited no significant reinstatement of preference, having a difference score that was significantly lower than that of the saline group (ANOVA, $F=5.08, P<0.05$ ) and did not differ from zero (Fig. 2C).

\section{Discussion}

Recent research has suggested that established drug-cue memories can be attenuated by pharmacological interventions. In particular, several experiments have shown that administration of neuropharmacological agents immediately before or after the retrieval of drug-cue memories leads to decreased expression of those memories on subsequent tests. An important question is whether these interventions truly interfere with memory for the drug-cue associations, or instead work through other associative processes, e.g., by facilitating extinction of the drug-cue memories. The process of facilitated extinction can be distinguished experimentally from reconsolidation interference because memories that are extinguished are subject to the possibility of spontaneous recovery, renewal, and reinstatement (Myers and Davis 2002).

The present findings allow us to distinguish between facilitated extinction and reconsolidation interference induced by post-retrieval administration of propranolol. In experiment 1 , the animals given a single post-retrieval injection of propranolol reached the no-preference criterion more quickly than those that received a saline injection (Fig. 1A,B). Once this criterion was met, both the propranolol- and saline-treated animals were given a cocaine priming injection and exhibited a robust reinstatement of preference (Fig. 1C). These results suggest that, although the single propranolol injection significantly affected reconsolidation of the memory, the memory was not abolished. Following post-test 1, it appears that the slightly weakened cocaine-context association of the propranolol group underwent normal extinction during the subsequent week, but the original memory persisted. This intact memory of the original association was used to guide reinstatement of seeking out the cocaine-paired context once the cocaine priming injection was administered.

In experiment 2 , animals received daily post-test injections of propranolol or saline. The propranolol group reached the nopreference criterion sooner than the saline group (Fig. 2A,B). This result is similar to, but more pronounced than, the group differences observed during experiment 1 . Contrary to what was seen in experiment 1 , the animals that received daily post-test propranolol injections during the unreinforced preference testing phase did not reinstate their preference for the drug-paired environment when given a cocaine priming injection. However, the saline group of experiment 2 lost their preference for the cocaine-paired compartment during $12 \mathrm{~d}$ of unreinforced preference testing and robustly reinstated that preference when given a cocaine challenge. The most parsimonious explanation of these findings is that the daily post-test propranolol injections de- 
A

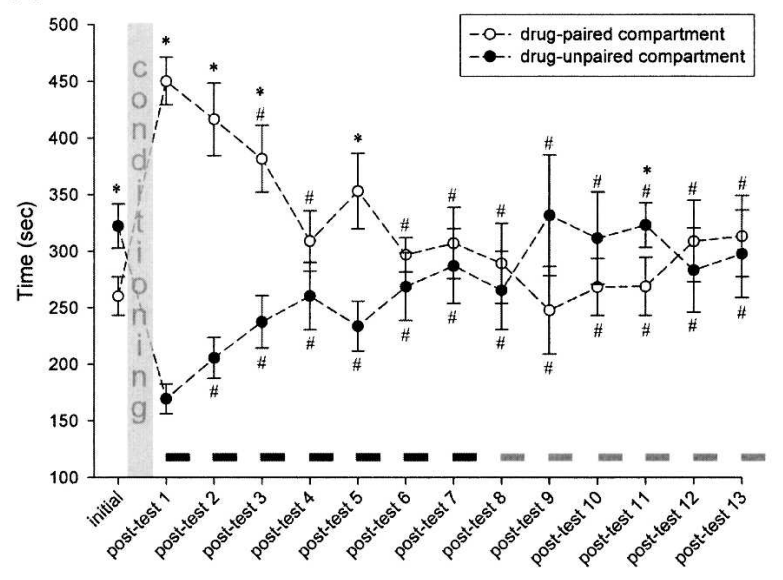

B

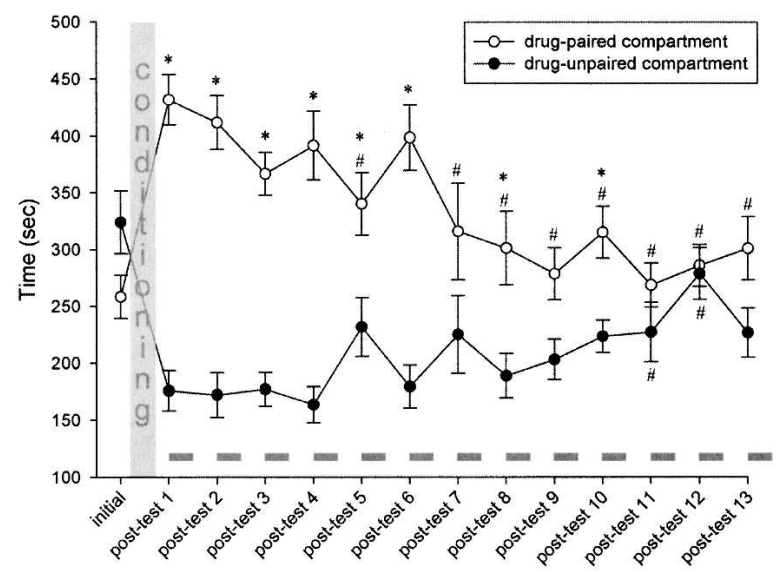

C

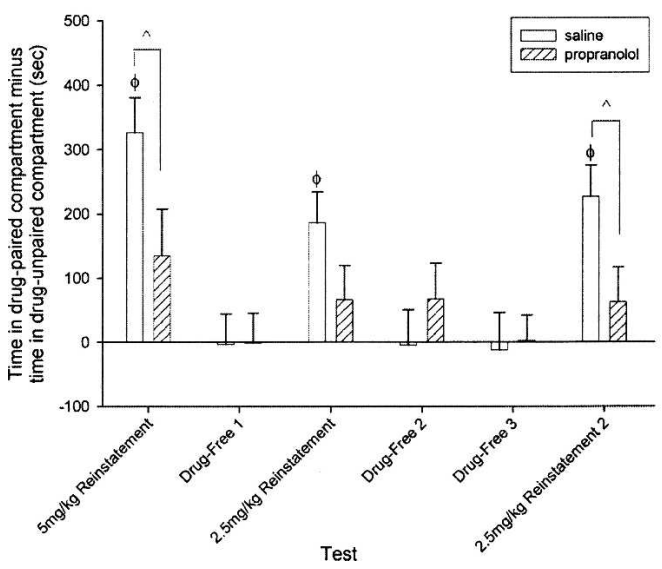

Figure 2. $(A, B)$ COC-CPP expression indicated by mean \pm S.E.M. time spent in the drug-paired vs. drug-unpaired compartments. (A) Propranolol group $(n=12)$, (B) saline group $(n=12)$. Black bars indicate occurrences of propranolol injections; gray bars indicate occurrences of saline injections. $\left(^{*}\right)$ Indicates significant difference between compartments, Wilcoxon tests, $P<0.05$. (\#) Indicates significant difference from posttest 1 , Wilcoxon tests, $P<0.05$. (C) COC-CPP expression following reinstatement indicated by time in the drug-paired compartment minus time in the drug-unpaired compartment. $\left({ }^{\wedge}\right)$ Indicates significant difference between groups, Wilcoxon tests, $P<0.05$. ( $\Phi)$ Indicates significant difference from zero, one-sample $t$-tests, $P<0.05$. Although not statistically significant, the first $2.5-\mathrm{mg} / \mathrm{kg}$ reinstatement test tended toward a difference between the groups $(P<0.1)$. creased the extent of the conditioned preference by cumulatively interfering with reconsolidation of the original memory, whereas the saline group underwent extinction. We cannot rule out the possibility that the propranolol animals in this experiment also underwent new extinction learning during the period of daily unreinforced preference testing, but the results of the cocaine priming tests reveal that the original cocaine-context associations did not guide the preferences of this group during the reinstatement tests. Consequently, the reconsolidation interference resulting from post-retrieval propranolol administrations appears to override any other influences.

An alternate explanation for these findings concerns the possible interaction of stress and propranolol. If the multiple injections experienced during experiment 2 contribute to a stress response of the saline group during the cocaine reinstatement phase, and if the propranolol treatments blunt the influence of this stress on subsequent reinstatement testing, then differential stress responses could have contributed to the group differences in the second experiment. However, stress appears unlikely to have been solely responsible for the differing levels of reinstatement observed in the propranolol and saline groups of experiment 2 , since (1) the propranolol group exhibited no significant reinstatement of preference following cocaine administration and (2) putative influences of stress carrying over from the multiple injection phase would be expected to result in reinstatement during the "drug free" testing days, yet no reinstatement in either group occurred.

Research using both fear-based and appetitive learning paradigms has shown that memory disruption during the labile postretrieval phase leads to decreased memory performance at later tests. Several different compounds, acting as antagonists or agonists of muscarinic, NMDA, and $\beta$-adrenergic receptors, administered either immediately before or after retrieval of cocaine-, amphetamine-, or morphine-cue associations, have been found to reduce later expression of those associations (Bernardi et al. 2006; Botreau et al. 2006; Popik et al. 2006; Kelley et al. 2007; Robinson and Franklin 2007; Sadler et al. 2007; Milton et al. 2008). However, the evidence concerning the effects of these compounds on drug-primed reinstatement of memory has been mixed. Kelley et al. (2007) reported that single administrations of scopolamine, MK-801, and d-cycloserine given to mice at the time of retrieval of a cocaine CPP reduced preference for the cocaine-paired compartment one week later, yet subsequent cocaine priming injections reinstated preference in all groups except that given MK-801. Similarly, Popik et al. (2006) found that administration of several compounds (chlordiazepoxide, lysergic acid diethylamide, or the NMDA antagonist memantine) at the time of retrieval of a morphine CPP reduced later preference for the morphine-paired compartment, yet subsequent morphine priming injections reinstated preference in all groups except that given memantine. Also, Robinson and Franklin (2007) report that single administration of propranolol at the time of reactivation of a morphine-contextual association disrupted later preference for that context yet did not affect morphine-induced reinstatement of the preference.

This recent literature motivates the conclusion that the ability of any compound administered at the time of retrieval of a drug-cue association to reduce later retention of that association is not sufficient evidence to demonstrate that the compound has interfered with reconsolidation of that memory. The possibility that a compound administered post-retrieval may also be accelerating extinction processes or interfering with retrieval processes merits investigation. One important distinction between the studies cited above and the present work is that here the reconsolidation interference was observed with repeated postretrieval administrations of propranolol. Supporting the interpre- 
tation that repeated administrations of pharmacological agents may cumulatively interfere with reconsolidation, Sadler et al. (2007) concluded that repeated post-retrieval administrations of MK-801 led to a disruption of an established amphetamine CPP that appeared independent of extinction processes. An additional factor that has been implicated in the lability of established CPP memories is the concomitant re-experience of both the context and the drug. Milekic et al. (2006) found that an established morphine CPP was disrupted following systemic injection of anisomycin or cycloheximide, as long as the injection followed re-exposure to both the context and morphine.

The present work supports earlier findings that postretrieval $\beta$-adrenergic receptor antagonism can decrease both cocaine conditioned place preference (Bernardi et al. 2006) and instrumental responding for cocaine-associated cues (Milton et al. 2008). This research extends the findings of earlier studies by demonstrating that this decrease is due to interference with reconsolidation of the original memory, not facilitation of extinction. Importantly, the results of Milton et al. (2008) appear unlikely to be explained by accelerated extinction. This group tested their animals for $29 \mathrm{~d}$ after propranolol administration in a reconsolidation-interference paradigm and reports no evidence of spontaneous recovery, which might have been expected if propranolol had facilitated extinction processes rather than interfered with memory.

Limitations to, and alternative explanations for, the current findings have been considered. It is possible that other nonassociative effects of propranolol are responsible for the lack of cocaine-primed reinstatement of preference; however, this seems unlikely. In their work, Milton et al. (2008) found that animals injected with propranolol, but denied a reactivation session, were unimpaired in their responding for cocaine-associated cues, suggesting that the propranolol had no nonspecific effects on lever pressing performance. Similarly, Robinson and Franklin (2007) showed that propranolol only disrupted memory for morphine CPP when given post-reactivation; control animals that received propranolol in their home cage were not impaired when tested for preference $24 \mathrm{~h}$ later. Sadler et al. (2007) found that rats receiving multiple, daily injections of MK-801 that were delayed by $60 \mathrm{~min}$ after each retrieval session showed no lasting impairments in amphetamine CPP. In aggregate, several preceding experiments have addressed the possibility that nonassociative effects of propranolol or other agents may be responsible for their subsequent effects on drug-cue preferences, and uniformly these studies have shown that preference is lost only when the drugs are given when the memories are reactivated.

Finally, the present results do not provide evidence concerning the neural locus of the effects of $\beta$-adrenergic receptor antagonists on reconsolidation of cocaine-contextual memories. The reconsolidation of fear memories depends upon the lateral amygdala (Debiec and Ledoux 2004; Debiec et al. 2006), and the interference with fear memory by post-retrieval administration of propranolol can be ascribed to its action in the amygdala (Debiec and Ledoux 2004). Together with evidence indicating the involvement of intracellular signaling pathways of the amygdala in the reconsolidation of cocaine-cue memories (Lee et al. 2005 , 2006), a strong argument can be made that effects observed in the present experiments depend upon the actions of propranolol within the basolateral amygdala; however, the hypothesis remains to be tested.

The experiments presented here outline a successful strategy for interfering with reconsolidation of drug-cue memories that depends upon interference with neurotransmitter receptors rather than with intracellular signaling pathways. The ability to distinguish reconsolidation interference from extinction of drugcue memories should aid in developing novel treatments for addiction. Although the facilitated extinction of drug-cue memories may prove to be of importance in the treatment of addiction, interfering with reconsolidation of the original drug-cue memories should provide a more powerful and enduring approach.

\section{Materials and Methods}

\section{Animals}

All animals were individually housed on a 12-h light/dark cycle with ad libitum access to food and water. The housing conditions and care of the animals were consistent with those specified in the "Guide for the Care and Use of Laboratory Animals" (National Research Council 1996). All procedures were performed during the light part of the diurnal cycle. Male Sprague-Dawley rats (Charles River Labs) weighing 275-300 g were handled for three days prior to behavioral conditioning.

\section{Place preference apparatus}

Conditioning took place in a three-chamber apparatus (Med Associates Inc.) consisting of two larger compartments $(11 \times 8.25$ inches) separated by a smaller compartment $(4.75 \times 8.25$ inches). The two larger compartments were distinguished by different visual, olfactory, and tactile cues. One compartment had white walls and a wire mesh floor above pine shavings. The other compartment had black and white checkered walls and a bar floor above cedar shavings. The middle compartment had a checkered wall and a white wall that led into the corresponding compartments as well as two gray walls and a solid gray PVC floor above the same corncob bedding as the home cage. The three compartments were separated by guillotine doors $(3.5 \times 4$ inches), patterned to match the outer compartments, which were lowered on conditioning days and raised on test days. The apparatus also contained a total of 15 photobeams that allowed for quantification of time spent in each of the three compartments during preference testing.

\section{Experiment 1: Single post-retrieval propranolol administration}

Baseline preferences were assessed by placing the animals in the center compartment of the place preference apparatus and allowing free access to all compartments for $15 \mathrm{~min}$. Time spent in each compartment was recorded. Because a Wilcoxon signedranks test revealed a statistically significant preference for the checkered compartment, we paired the cocaine injections with the white compartment. Conditioning took place over the next 4 d. Animals were divided into two groups such that each group had an equal mean time spent in each compartment. On each of the conditioning days animals received an injection of either cocaine-HCl (12 mg/kg, i.p.; Sigma) or saline $(1 \mathrm{~mL} / \mathrm{kg}$, i.p. $)$ and were immediately confined to the respective compartment of the apparatus for $30 \mathrm{~min}$. A counterbalanced design was used so that half of the animals were given cocaine prior to placement in the white compartment on the first conditioning day, and half received saline prior to placement in the checkered compartment on that day. The injections were then alternated for subsequent conditioning sessions.

Forty-eight hours after the last conditioning session, preference was assessed in a manner identical to that used to test baseline preferences (post-test 1). Immediately following their preference test and removal from the apparatus, animals received an injection of either propranolol-HCl $(10 \mathrm{mg} / \mathrm{kg}$, s.c.; Sigma; $n=10)$ or saline ( $1 \mathrm{~mL} / \mathrm{kg}$, s.c.; $n=12)$ and were returned to their home cage. The following day these animals were again tested for their preference (post-test 2), marking the beginning of an unreinforced preference testing protocol comprised of one unreinforced preference test per day. This protocol was continued until both groups reached a "no-preference criterion." This criterion was defined as two consecutive days of testing during which (1) the total time spent in each compartment did not differ significantly and (2) the mean time spent by the group in the drug- 
paired compartment did not exceed the time spent in the drugunpaired compartment by $>90 \mathrm{sec}$.

Twenty-four hours after the no-preference criterion was reached by both groups (post-test 10), all animals received a priming injection of cocaine $(5 \mathrm{mg} / \mathrm{kg}$, i.p.) immediately prior to a preference test. To assess the longevity of the cocaine-primed reinstatement, on each of the following two days the animals underwent preference tests while drug-free.

\section{Experiment 2: Multiple post-retrieval propranolol administrations}

Baseline preference testing and conditioning were performed as described in experiment 1. Forty-eight hours after the last conditioning session, animals were given a preference test (post-test $1)$ and received either a propranolol $(10 \mathrm{mg} / \mathrm{kg}$, s.c.; $n=12)$ or saline $(1 \mathrm{~mL} / \mathrm{kg}$, s.c.; $n=12)$ injection immediately afterward. On subsequent days, the animals continued to receive one preference test per day followed by either a propranolol or saline injection until the no-preference criterion was reached by the propranolol group (post-test 7). After the propranolol-treated rats reached this criterion, those animals were then switched to posttest saline injections, and the one test per day protocol was continued for all animals until the saline group reached the nopreference criterion (post-test 12). Forty-eight hours after the saline group reached this criterion, both groups received a priming injection of cocaine $(5 \mathrm{mg} / \mathrm{kg}$, i.p.) immediately prior to a preference test. The next day, the animals underwent a drug-free preference test. Twenty-four hours later, a lower dose priming injection of cocaine $(2.5 \mathrm{mg} / \mathrm{kg}$, i.p.) was given immediately prior to preference testing in order to assess the possibility of a dosespecific effect on reinstatement. On each of the next two days, the animals again underwent drug-free preference testing. On the final day, the animals again received the lower dose priming injection $(2.5 \mathrm{mg} / \mathrm{kg}$, i.p.) immediately before a preference test.

\section{Statistics}

Wilcoxon signed-ranks tests were conducted to compare the amount of time spent in the drug-paired and drug-unpaired compartments at each test, as well as to compare time spent in the drug-paired compartment across tests within treatment groups. Repeated measures ANOVAs were used to evaluate group differences in preference across tests. One-sample $t$-tests were used to compare difference scores against zero. Differences with $P$-values $<0.05$ were considered statistically significant.

\section{Acknowledgment}

We acknowledge a PHS grant (DA 021807) to J.F.M.

\section{References}

Bernardi, R.E., Lattal, K.M., and Berger, S.P. 2006. Postretrieval propranolol disrupts a cocaine conditioned place preference. Neuroreport 17: 1443-1447.

Botreau, F., Paolone, G., and Stewart, J. 2006. d-Cycloserine facilitates extinction of a cocaine-induced conditioned place preference. Behav. Brain Res. 172: 173-178.

Childress, A., Ehrman, R., McLellan, A.T., and O’Brien, C. 1988. Conditioned craving and arousal in cocaine addiction: A preliminary report. NIDA Res. Monogr. 81: 74-80.

Debiec, J. and Ledoux, J.E. 2004. Disruption of reconsolidation but not consolidation of auditory fear conditioning by noradrenergic blockade in the amygdala. Neuroscience 129: 267-272.

Debiec, J., Doyere, V., Nader, K., and Ledoux, J.E. 2006. Directly reactivated, but not indirectly reactivated, memories undergo reconsolidation in the amygdala. Proc. Natl. Acad. Sci. 103: 3428-3433.

Delamater, A.R. 2004. Experimental extinction in Pavlovian conditioning: Behavioural and neuroscience perspectives. Q. J. Exp. Psychol. B 57B: 97-132.

Diergaarde, L., Schoffelmeer, A.N., and De Vries, T.J. 2006. $\beta$-Adrenoceptor mediated inhibition of long-term reward-related memory reconsolidation. Behav. Brain Res. 170: 333-336.

Duvarci, S. and Nader, K. 2004. Characterization of fear memory reconsolidation. J. Neurosci. 24: 9269-9275.

Kelley, J.B., Anderson, K.L., and Itzhak, Y. 2007. Long-term memory of cocaine-associated context: Disruption and reinstatement. Neuroreport 18: 777-780.

Lee, J.L., Everitt, B.J., and Thomas, K.L. 2004. Independent cellular processes for hippocampal memory consolidation and reconsolidation. Science 304: 839-843.

Lee, J.L., Di Ciano, P., Thomas, K.L., and Everitt, B.J. 2005. Disrupting reconsolidation of drug memories reduces cocaine-seeking behavior. Neuron 47: 795-801.

Lee, J.L., Milton, A.L., and Everitt, B.J. 2006. Cue-induced cocaine seeking and relapse are reduced by disruption of drug memory reconsolidation. J. Neurosci. 26: 5881-5887.

Milekic, M.H., Brown, S.D., Castellini, C., and Alberini, C.M. 2006. Persistent disruption of an established morphine conditioned place preference. J. Neurosci. 26: 3010-3020.

Miller, C.A. and Marshall, J.F. 2005. Molecular substrates for retrieval and reconsolidation of cocaine-associated contextual memory. Neuron 47: 873-884.

Milton, A.L., Lee, J.L., and Everitt, B.J. 2008. Reconsolidation of appetitive memories for both natural and drug reinforcement is dependent on $\beta$-adrenergic receptors. Learn. Mem. 15: 88-92.

Mueller, D. and Stewart, J. 2000. Cocaine-induced conditioned place preference: Reinstatement by priming injections of cocaine after extinction. Behav. Brain Res. 115: 39-47.

Myers, K.M. and Davis, M. 2002. Behavioral and neural analysis of extinction. Neuron 36: 567-584.

National Research Council 1996. Guide for the care and use of laboratory animals. National Academy Press, Washington, D.C.

Pavlov, I.P. 1927. Conditioned reflexes. Oxford University Press, London.

Popik, P., Wrobel, M., and Bisaga, A. 2006. Reinstatement of morphine-conditioned reward is blocked by memantine. Neuropsychopharmacology 31: 160-170.

Robinson, M.J. and Franklin, K.B. 2007. Central but not peripheral $\beta$-adrenergic antagonism blocks reconsolidation for a morphine place preference. Behav. Brain Res. 182: 129-134.

Sadler, R., Herzig, V., and Schmidt, W.J. 2007. Repeated treatment with the NMDA antagonist MK-801 disrupts reconsolidation of memory for amphetamine-conditioned place preference. Behav. Pharmacol. 18: $699-703$.

Sara, S.J. 2000. Retrieval and reconsolidation: Toward a neurobiology of remembering. Learn. Mem. 7: 73-84.

Stewart, J. 1983. Conditioned and unconditioned drug effects in relapse to opiate and stimulant drug self-administration. Prog. Neuropsychopharmacol. Biol. Psychiatry 7: 591-597.

Taubenfeld, S.M., Milekic, M.H., Monti, B., and Alberini, C.M. 2001. The consolidation of new but not reactivated memory requires hippocampal C/EBPß. Nat. Neurosci. 4: 813-818.

Received April 28, 2008; accepted in revised form June 25, 2008. 


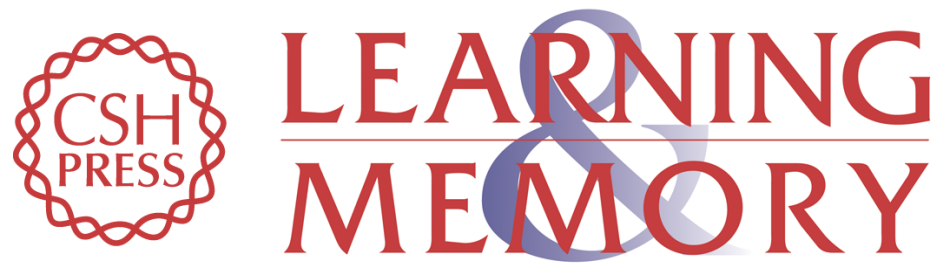

\section{Post-retrieval $\beta$-adrenergic receptor blockade: Effects on extinction and reconsolidation of cocaine-cue memories}

Ashley N. Fricks-Gleason and John F. Marshall

Learn. Mem. 2008, 15:

Access the most recent version at doi:10.1101//m.1054608

References This article cites 23 articles, 7 of which can be accessed free at: http://learnmem.cshlp.org/content/15/9/643.full.html\#ref-list-1

License

Email Alerting

Receive free email alerts when new articles cite this article - sign up in the box at the Service top right corner of the article or click here. 